\title{
Electron transport through nanosystems driven by pseudo-Gaussian well scattering
}

\author{
Felix Iacob \\ West University of Timişoara, 300223 V. Pârvan 4, Timişoara, Romania \\ felix.iacob@gmail.com
}

PACS 61.46.w, 34.80.i

DOI 10.17586/2220-8054-2020-11-1-44-49

Electron transmission through nanosystems encounter different scattering processes. We focus on the scattering by impurities, which were implemented by considering a model based on the pseudo-Gaussian well. We discuss typical signatures of this phenomenon.

Keywords: Structure of nanoscale materials, electron scatter.

Received: 12 January 2020

\section{Introduction}

Particle scattering outside the target has been one of the most important applications of quantum mechanics. Probably, it is the most efficient way to study the structure of matter at short distances. A high-momentum scattering is required, by uncertainty principle, in order to achieve better resolutions. However, a low energy study reveals the excitation spectrum of a system, the resonances. Particle scattering is of great interest in disruptive technologies, as it critically depends on the dissipationless transport of electrons. It is of great interests about electron mobility calculation as well as the specific equations for the different scattering mechanisms [1]. This allows the evaluation of properties at the macro level, as well as the evaluation of measure at the nanoscale voltage drop caused by the scattering was previously reported [2].

In this work we, investigate the electrons coming in and getting scattered by a short-ranged potential, namely, the pseudo-Gaussian potential [3-5] considered to be located around the origin. Nanosystems having size approaching the fundamental scale of microscopic length have fundamentally demonstrated new physical phenomena. New advances have been made in many basic and modified fields of nanophysics, including diluted cold gases, carbon nanotubes, graphene, magnetic nanostructures, composite nanoparticles, transport through coupled quantum dots, spin-dependent electron transport phenomena, optical arrays with doped nanoparticles, and electronic, molecular, and quantum information processing. The pseudo-Gaussian potential proves to be a reliable model for defects in nanostructures or a bulk material itself. It can easily model the vibrational levels of such a nanostructure based on the fact it has a finite levels of energies, as shown in different papers as [6,7], despite of harmonic oscillator with an infinite one. Moreover, change of number of levels is possible by manipulation of some parameters, and also it permits dissociation with the energy in the continuum. Heterostructures can be used for advanced electronic devices (for example, modulated field effect transistors, heterojunction bipolar transistors, and resonant tunneling devices), optical components (e.g., waveguides, mirrors) and optoelectronic devices and structures (for example, laser diodes, photodetectors). These are also crucial in many optoelectronic devices (e.g., lasers). Perhaps their most important technological aspect may be that they can be used for all these electronic, optical and optoelectronic purposes and, therefore, can allow the integration of all of them. The scattering method of investigation can be found in any area of physics even in astrophysics [8].

\section{Pseudo-Gaussian oscillator model}

The computation of the scattering data for a given potential requires the construction of the regular solution of the radial equation. We, shortly, introduce some aspects of the pseudo-Gaussian oscillator (PGO) model. The central real valued potential:

$$
V_{\lambda, \mu}^{s}(r)=\left(\lambda+\sum_{k=1}^{s} C_{k} r^{2 k}\right) \exp \left(-\mu r^{2}\right),
$$

is fixed by the coefficients $C_{k}$ :

$$
C_{k}=\frac{(\lambda+k) \mu^{k}}{k !}
$$

and its properties are completely determined by the dimensionless parameters $\lambda \in \mathbb{R}, \mu \in \mathbb{R}_{+}$and the positive integer $s=1,2, \ldots$, named the order of PGO. The potentials defined by the Eqs. (1) and (2) have the property to approach to the HO potential when $r \rightarrow 0$ together with Gaussian asymptotic behavior, i.e. $\lim _{r \rightarrow \infty} V_{\lambda, \mu}^{s}(r)=0$. The plot from 
Fig. 1, shows the shape of PGO poyential. A last observation on the potential is that the Taylor expansion does not have terms proportional with $r^{4}, r^{6}, \ldots, r^{2 s}$, for a fixed $s$,

$$
V_{\lambda, \mu}^{s}(r)=\lambda+\mu r^{2}+\sum_{k=s+1} \hat{C}_{k} r^{2 k} .
$$

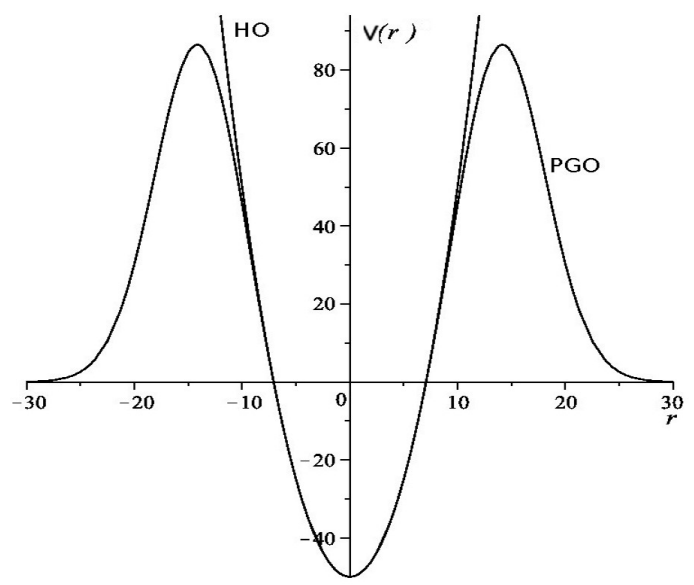

FIG. 1. The pseudo-Gaussian oscillator potential graph $(s=3)$ compared with harmonic oscillator potential one

The evolution in this potential is given by Schrödinger equation:

$$
\left[\frac{\mathrm{d}^{2}}{\mathrm{dr}^{2}}+(E-V(r))-\frac{l(l+1)}{r^{2}}\right] R(r, t)=-\frac{i \hbar}{2 \pi} \frac{\partial R(r, t)}{\partial t},
$$

where $\psi(r, t) \equiv r^{-1} R(r, t)$ eliminates the first derivative. It was shown [4] that the time independent equation of (4) generates a stationary states in the pseudo-Gaussian well,

$$
R(r)=\exp [p(r)] \sum_{n=0} a_{n} r^{2 n+\tau},
$$

the polynomial $p(r)$ has the form:

$$
p(r)=\sum_{n=1}^{s+1} \frac{1}{2 n} \alpha_{2 n} r^{2 n},
$$

where $\alpha_{2 n}$ are parameters. The analytic expression of ground state looks like:

$$
\psi_{0}(r)=a_{0} \exp \left[0.638 r^{2}-0.039 r^{4}+0.0034 r^{6}-0.0029 r^{8}\right] .
$$

A plot of wave eigenfunctions for $n \in\{0,1,2,3\}$ is shown in Fig. 2.

To evaluate the transport of electron through a nanostructure where impurities are supposed to be modeled with PG potential, we notice that it is independent on the polar and azimuth coordinate $(\theta, \phi)$. The probability of current $J(r, t)$ :

is defined:

$$
J(r, t)=\frac{i}{2 m}\left(\psi \frac{\partial \psi^{*}}{\partial r}-\psi^{*} \frac{\partial \psi}{\partial r}\right)
$$

$$
\frac{\mathrm{dP}_{\mathrm{ab}}(\mathrm{t})}{\mathrm{dt}}=J(a, t)-J(b, t),
$$

such that describes the change in the probability of finding a particle in the region bordered by a and $\mathrm{b}$ at the time $\mathrm{t}$. In order to overcome the problems caused due to the centrifugal barrier we organize ourselves completing a procedure regarding the scattering amplitude. The scattering amplitude $f\left(k, k^{\prime}\right)$ solution of the time independent equation (4), corresponding to the particle incident on the scattering centre in the solid angle $\Omega(\theta, \phi)$, has the form, [9]:

$$
f\left(k, k^{\prime}\right)=-\frac{m}{\sqrt{2 \pi}} \int \mathrm{dr}^{\prime} \mathrm{e}^{-\mathrm{ikr} \mathrm{r}^{\prime}} \mathrm{V}\left(\mathrm{r}^{\prime}\right) \psi_{\mathrm{n}}\left(\mathrm{r}^{\prime}\right)
$$

where a Born approximation is not required, due to the wave function which has the known expression (5). 


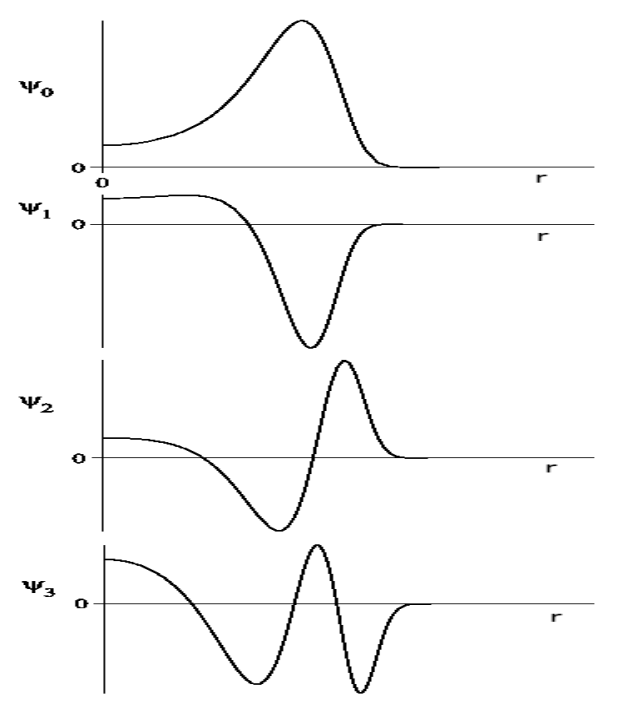

FIG. 2. The first four eigenfunctions for pseudo-Gaussian oscillator, $N=7, \lambda=-5.6, \mu=0.2$ and $l=0$

The current (8) has the form:

$$
J_{\text {scat }}=\frac{r^{2}}{2 m i(2 \pi)^{3}}\left|f\left(k, k^{\prime}\right)\right|^{2}\left[\frac{\mathrm{e}^{-\mathrm{ikr}}}{r} \partial_{r} \frac{\mathrm{e}^{\mathrm{ikr}}}{r}+c . c .\right]
$$

Finally, we get that the cross section has the expression,

$$
\frac{\mathrm{d} \sigma}{\mathrm{d} \Omega}=\left|f\left(k, k^{\prime}\right)\right|^{2},
$$

knowing that the probability flux associated with the incident wavefunction is $j_{i n c}=\frac{\hbar}{m(2 \pi)^{3}} k$ [13].

\section{Results of calculations and discussion}

Based on the previous section were performed calculations of electron scattered on the PGO structure, which simulates conduction resistivity in nanostructure. The explicit formulae calculation ia given in Appendix (A). The main difficulties that had to be overcome were due to centrifugal barrier and the fact that even if equation (4) is solvable the potential is an infinite series, thus just an formal solution of (4) can be given. In order to obtain specific computation firstly the potential have to be finite, i.e. to specify the depth of the well. We performed our computation with the potential (13), by setting $s=4$, similar computation can be made for any $s$, as was shown [4]. This is straightforward due to the Gaussian integrals, (17), that are generated by the infinite series of potential and which can be solved for each term taken as a sum of integrals. This observation facilitates a direct calculation of the scattering amplitude, the cross-section and so on. The calculation considers the following constant values: Planck constant $h=4.13566766 \mathrm{eV} \cdot \mathrm{s}$, electron mass $m=0.511 \mathrm{MeV}$, and provides a treatment for an electron collisions with PGO scattering potential. Fig. 3, schematically represents the associated plane wave, the free particle wave function can

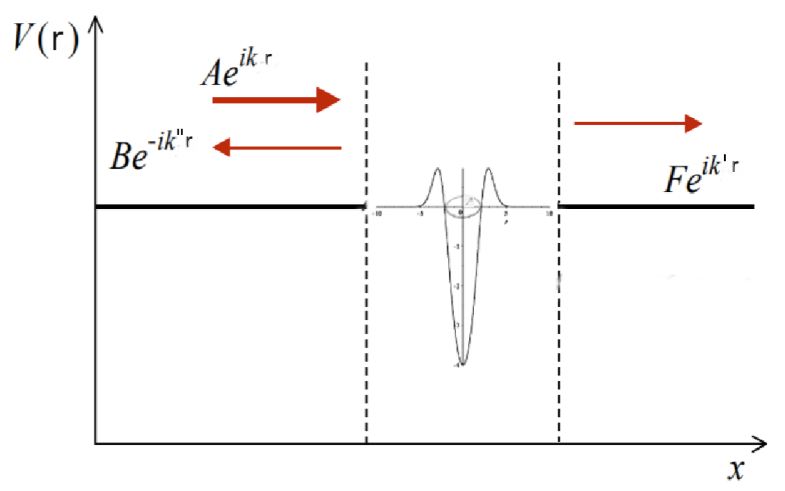

FIG. 3. Plan wave scatter on pseudo-Gaussian oscillator, $N=4, \lambda=-4, \mu=0.2$ 
be represented by a superposition of momentum eigenfunctions, with coefficients given by the Fourier transform of the plane wave, representing the wave packet. The interaction in the potential region is given by a series of Gaussian integrals. The work is facilitated by the fact that the PGO potential has the Taylor expansion simplified by the fact that the terms proportional with $r^{4}, r^{6}, \ldots, r^{2 s}$, are missing for a given $s$, as (3) shows. Making use of Gaussian integral properties, appendix relations (16) and (17), the scattering amplitude, (10), was obtained as it is given in the appendix expression (14). With the use of latter mentioned expression, it was given the expression to the cross section (15).

In the Fig. 4, one can see different cross sections calculated for certain particular cases of potentials. In all cases, it can be observed a resonant state given by the oscillatory structure. As one can compare with the resonance disposal in works like ( [10-12]), it not belongs to the outer-well resonances. For a shallow deep potential endowed with high barriers, Fig. 4(b), extra resonances can be observed with a low probability, Fig. 4(a). As the potential gets deeper and barriers decrease, Fig. 4(d), the extra resonances reduces, Fig. 4(c). Finally, for a well without barrier, Fig. 4(f), the cross section, Fig. 4(e), does not exhibit resonant states. In this way, a model with a barriers can be used to explain the electronic conductance through nanostructure. The resistive transition is caused due to the resonant capture of passing

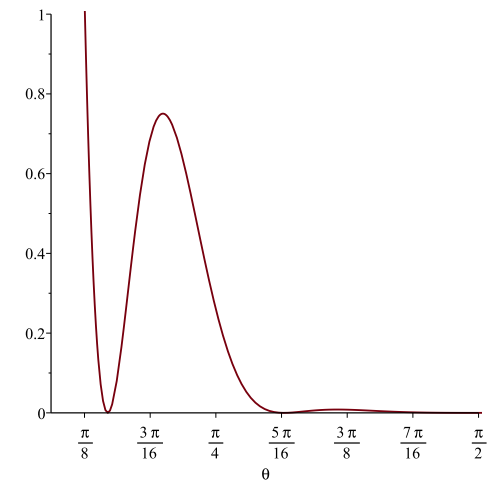

(A) cross section, $\lambda=-4, \mu=0.1$

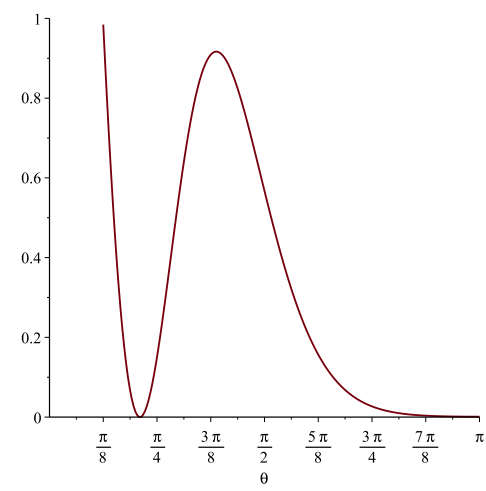

(C) cross section, $\lambda=-4, \mu=0.5$

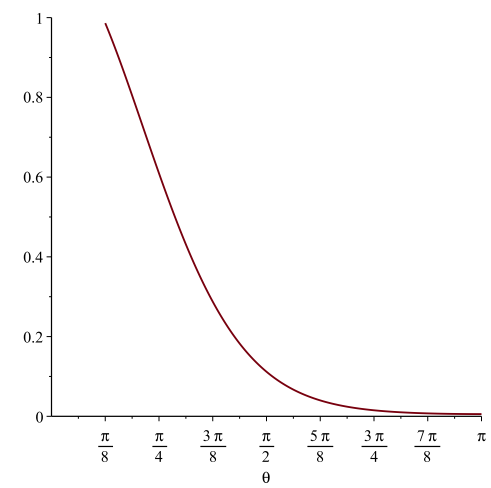

(E) cross section, $\lambda=-4, \mu=0.9$

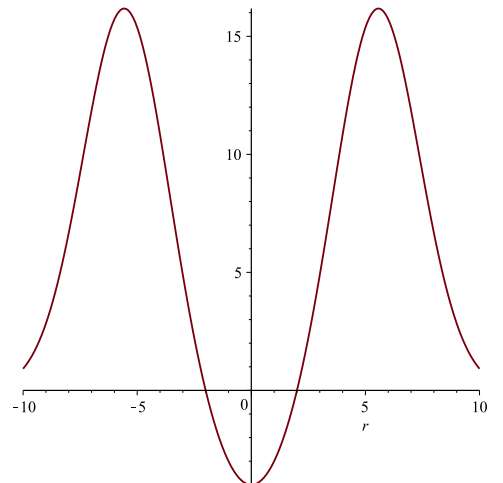

(B) potential

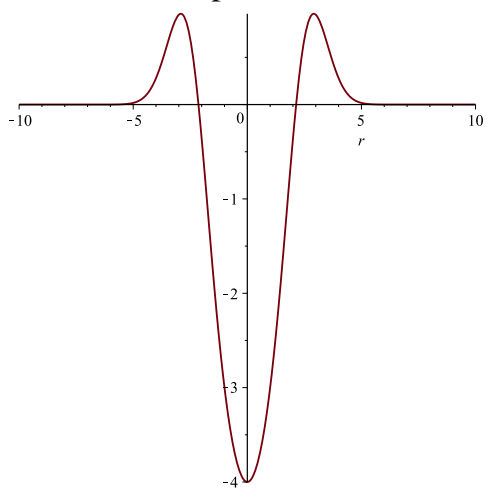

(D) potential

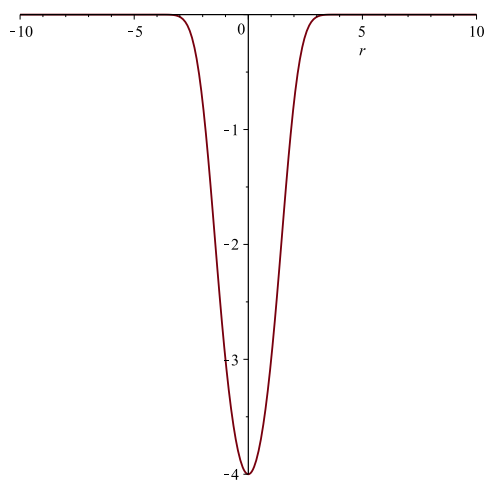

(F) potential

FIG. 4. The cross section, on left, vs. shape of potential corresponds to, on right 
electron along the impurity described by the PGO. Our calculation shows that Rydberg states cannot be formed, being in the case of Fig. 4(e).

In conclusion the PGO model applied to electronic transport in nanostructure confirm that a part of resistivity is given by scattering on impurity centers, described by PGO potential, caused by capture of electron in a resonant state. The resonant state is confirmed on a narrow potential center, $\mu=0.1$, and barriers whose size is comparable to the wavelength of the electron and disappears with increasing of the potential size.

\section{Acknowledgements}

The author like to acknowledge the COST Action CA17126, Towards understanding and modelling intense electronic excitation, part of this is due to the support for collaboration and discussions established with other scientists through the network.

\section{References}

[1] Sadi T., Medina-Bailon C., et al. Simulation of the Impact of Ionized Impurity Scattering on the Total Mobility in Si Nanowire Transistors. Materials, 2019, 12 (1), 124.

[2] Bauer S., Bobisch C.A. Nanoscale electron transport at the surface of a topological insulator. Nat. Commun., $2016,7,11381$.

[3] Iacob F. Relativistic pseudo-Gaussian oscillators. Phys. Lett. A, 2010, 374 (11-12), P. 1332-1335.

[4] Iacob F., Lute M. Exact solution to the Schrodingers equation with pseudo-Gaussian potential. J. Math. Phys., 2015, 56, 121501.

[5] Iacob F. Spectral Characterization Of Hydrogen-Like Atoms Confined By Oscillating Systems. Centr. Eur. J. Phys., 2014,12 (9), P. 628-636.

[6] Kuemmeth F., Bolotin K.I., Shi S.-F., Ralph D.C. Measurement of discrete energy-level spectra in individual chemically synthesized gold nanoparticles. Nano Lett., 2008, 8, 4506.

[7] Popov D., Dong S.H., et al. Construction of the Barut-Girardello quasi coherent states for the Morse potential. Annals of Physics, 2013, 339, P. 122-134.

[8] Cotăescu I.I., Sporea C.A. Scattering of Dirac fermions by spherical massive bodies. Eur. Phys. J. C, 2019,79 (1), 15.

[9] Johnson C.W. A practical introduction to quantum scattering theory, and beyond, 2017.

[10] Niyonzima S., Pop N., et al. Low-energy collisions between electrons and BeD+. Plasma Sources Science and Technology, 2018, 27 (2), 025015.

[11] Iacob F., Pop N., et al. Recombination and excitation of molecular cations with electrons: Application to BeD+ and BeT+. AIP Conference Proceedings, 2019, 2071 (1), 020007.

[12] Pop N., Iacob F., et al. Reactive collisions of electrons with HD+, BeH+, BeD+ and SH+. AIP Conference Proceedings, 2017 , 1916 (1), 020013.

[13] Fitzpatrick R. Scattering Theory. URL: https://farside.ph.utexas.edu/teaching/qm/lectures/node82.html.

\section{Appendix}

\section{A. The scattered amplitude and cross section expressions}

In our calculations we consider the potential (1) having the depth given by the chosen value of $s=4$,

$$
\begin{aligned}
V(r) & = \\
& {\left[\lambda+(\lambda \mu+1) r^{2}+(1 / 2 \lambda \mu+1) \mu r^{4}+\right.} \\
& \left.(1 / 6 \lambda \mu+1 / 2) \mu^{2} r^{6}+(1 / 24 \lambda \mu+1 / 6) \mu^{3} r^{8}\right] \mathrm{e}^{-\mu r^{2 \prime}},
\end{aligned}
$$

which gives the scattered amplitude,

$$
\begin{aligned}
f(q) & =-7.76 \times 10^{20} \frac{\sqrt{2 \pi}}{q}\left(\mathrm{e}^{1 / 4 \frac{q^{2}}{\mu}}\right)^{-1} \\
& {\left[1 / 4 \frac{\lambda \sqrt{2} q}{\sqrt{\pi} \mu} \sqrt{\frac{\pi}{\mu}}-1 / 16 \frac{\lambda \sqrt{2} q\left(q^{2}-6 \mu\right)}{\mu^{5 / 2}} 1 / 16 \frac{\sqrt{2} q\left(q^{2}-6 \mu\right)}{\mu^{7 / 2}}\right.} \\
& +\frac{\lambda \sqrt{2} q\left(q^{4}-20 \mu q^{2}+60 \mu^{2}\right)}{128 \mu^{7 / 2}}+\frac{\sqrt{2} q\left(q^{4}-20 \mu q^{2}+60 \mu^{2}\right)}{64 \mu^{9 / 2}} \\
& -\frac{\lambda \sqrt{2} q\left(q^{6}-42 \mu q^{4}+420 m u^{2} q^{2}-840 \mu^{3}\right)}{1536 \mu^{9 / 2}}-\frac{\sqrt{2} q\left(q^{6}-42 \mu q^{4}+420 \mu^{2} q^{2}-840 \mu^{3}\right)}{512 \mu^{11 / 2}} \\
& +\frac{\lambda \sqrt{2} q\left(q^{8}-72 \mu q^{6}+1512 \mu^{2} q^{4}-10080 \mu^{3} q^{2}+15120 \mu^{4}\right)}{24576 \mu^{11 / 2}} \\
& \left.+\frac{\sqrt{2} q\left(q^{8}-72 \mu q^{6}+1512 \mu^{2} q^{4}-10080 \mu^{3} q^{2}+15120 \mu^{4}\right)}{6144 \mu^{13 / 2}}\right]
\end{aligned}
$$


conversion to wave vectors $\sqrt{2 k^{2}(1-\cos (\theta))}$ cross section $(|(f(q))|)^{2}$ gives

$$
\begin{aligned}
\frac{\mathrm{d} \sigma}{\mathrm{d} \Omega}= & 3.21 \times 10^{36} \frac{1}{\mu^{13}}\left(\mathrm{e}^{1 / 2} \frac{k^{2}(-1+\cos (\theta))}{\mu}\right)^{-2} \\
& C^{2} \mid-8712.0 k^{2} \mu^{3}-168.0 k^{6} \mu+2112.0 k^{4} \mu^{2}+4.0 k^{8}(\cos (\theta))^{4} \\
& -16.0 k^{8}(\cos (\theta))^{3}+24.0 k^{8}(\cos (\theta))^{2}-16.0 k^{8} \cos (\theta)+3465.0 \lambda \mu^{5} \\
& +168.0 k^{6}(\cos (\theta))^{3} \mu-504.0 k^{6}(\cos (\theta))^{2} \mu+2112.0 k^{4}(\cos (\theta))^{2} \mu^{2} \\
& +504.0 k^{6} \cos (\theta) \mu-4224.0 k^{4} \cos (\theta) \mu^{2}+8712.0 k^{2} \cos (\theta) \mu^{3}+k^{8} \lambda \mu \\
& -2772.0 \lambda k^{2} \mu^{4}+594.0 \lambda k^{4} \mu^{3}-44.0 \lambda k^{6} \mu^{2}+\lambda k^{8}(\cos (\theta))^{4} \mu \\
& -4.0 \lambda k^{8}(\cos (\theta))^{3} \mu+44.0 \lambda k^{6}(\cos (\theta))^{3} \mu^{2}+6.0 \lambda k^{8}(\cos (\theta))^{2} \mu \\
& -132.0 \lambda k^{6}(\cos (\theta))^{2} \mu^{2}-4.0 \lambda k^{8} \cos (\theta) \mu+594.0 \lambda k^{4}(\cos (\theta))^{2} \mu^{3} \\
& +132.0 \lambda k^{6} \cos (\theta) \mu^{2}-1188.0 \lambda k^{4} \cos (\theta) \mu^{3}+2772.0 \lambda k^{2} \cos (\theta) \mu^{4} \\
& +4.0 k^{8}+\left.8316.0 \mu^{4}\right|^{2}
\end{aligned}
$$

substitution $\left(\lambda=-4, \mu=0.5, C=1.5 \times 10^{-44}, k=2\right)$.

\section{B. Gaussian integrals}

$$
\int_{0}^{\infty} r \sin (q r)\left(r^{2} \mathrm{e}^{-\mu r^{2}}\right) \mathrm{d} r
$$

gives

The influence of the centrifugal barrier:

$$
-1 / 16 \frac{\sqrt{\pi} q^{3}}{m u^{7 / 2}} \frac{1}{\sqrt[4]{\mathrm{e}^{\frac{q^{2}}{m u}}}}+3 / 8 \frac{\sqrt{\pi} q}{m u^{5 / 2}} \frac{1}{\sqrt[4]{\mathrm{e}^{\frac{q^{2}}{m u}}}} .
$$

$$
\lim _{n \rightarrow \infty} \int_{0}^{n} \frac{\sin (q r) l(l+1) \mathrm{e}^{-0.5 r^{2}}}{r} \mathrm{~d} r
$$

is an amortized oscillation as increasing the integration domain.

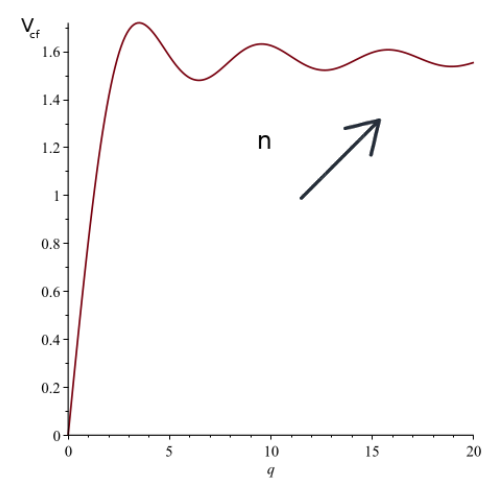

FIG. 5. The dependence on centrifugal barrier. As $n$, the domain, extends upwards the sinusoids become dense 